\title{
ELLIPTIC RIESZ OPERATORS ON THE WEIGHTED SPECIAL ATOM SPACES
}

\author{
KUANG JICHANG \\ Department of Mathematics \\ Hunan Normal University \\ Changsha, Hunan, 410081 \\ P.R. CHINA
}

(Received July 14, 1993 and in revised form March 3, 1995)

ABSTRACT. In this paper we study the boundedness and convergence of $\sigma_{r}^{s}(f)$ and $\tilde{\sigma}_{r}^{s}(f)$, the elliptic Riesz operators and the conjugate elliptic Riesz operators of order $s>0$, on the weighted special atom space $B(\omega)$.

KEY WORDS AND PHRASES. Elliptic Riesz operators, weighted special atom space, Lorentz spaces.

1991 AMS SUBJECT CLASSIFICATION CODES. 42B30, 42B08.

\section{INTRODUCTION.}

Let $R^{n}$ be $n$-dimensional Euclidean space and $Z^{n}$ be the unit lattice in $R^{n}$. The $n$-Torus $T^{n}$ is the coset space $R^{n} /\left(2 \pi Z^{n}\right), Q^{n}=\left\{x=\left(x_{1}, . ., x_{n}\right): 0<x_{k} \leq 2 \pi, 1 \leq k \leq n\right\}$. Let $A(D)$ be a selfadjoint elliptic differential operator with real coefficients defined on $C_{0}^{\infty}\left(R^{n}\right), A(D)=\sum_{|\alpha|=m} a_{\alpha} D^{\alpha}$, where $D^{\alpha}=\partial^{|\alpha|} / \partial x_{1}^{\alpha_{1}} \ldots \partial x_{n}^{\alpha_{n}}, \alpha=\left(\alpha_{1}, \ldots, \alpha_{n}\right)$ is multi index and $|\alpha|=\alpha_{1}+\ldots+\alpha_{n}$. We always assume that the set $\left\{x \in R^{n}: A(x)<1\right\}$ is convex and its boundary has non-vanishing Gaussian curvature everywhere.

The elliptic Riesz operators and the conjugate elliptic Riesz operators of order $s>0$ are defined respectively by

$$
\begin{gathered}
\sigma_{r}^{s}(f, x)=\sum_{m \in Z^{n}}(1-A(m / r))_{+}^{s} \widehat{f}(m) e^{\imath m x} \\
\tilde{\sigma}_{r}^{s}(f, x)=\sum_{m \in Z^{n}}(1-A(m / r))_{+}^{s} \widehat{f}(m) \widehat{K}(m) e^{i m x}
\end{gathered}
$$

where

$$
\widehat{f}(m)=(2 \pi)^{-n} \int_{Q^{n}} f(x) e^{-\imath m x} d x
$$

are the multiple Fourier coefficients of $f, K(x)=P(x) /|x|^{n+m}(x \neq 0)$ is a kernel with a homogeneous and harmonic polynomial $P(x)$ of order $m$, and $\tilde{f}$ is the conjugate function of $f$ with respect to the kernel $K(x) . \beta_{+}=\max \{0, \beta\}$. If $A(\xi)=|\xi|^{2}, \sigma_{r}^{s}(f), \tilde{\sigma}_{r}^{s}(f)$ is just the usual Bochner-Riesz mean.

The maximal elliptic Riesz operators defined by

$$
\sigma^{s}(f, x)=\sup _{r>0}\left|\sigma_{r}^{s}(f, x)\right|, \tilde{\sigma}^{s}(f, x)=\sup _{r>0}\left|\tilde{\sigma}_{r}^{s}(f, x)\right| .
$$

In this paper, using the weighted special atom space $B(\omega)$, we will study the boundedness and convergence of $\sigma_{r}^{s}(f)$ and $\tilde{\sigma}_{r}^{s}(f)$ for all $s>0$ and $n=1$. 
We rewrite $B(\omega)$ which was introduced in [4]:

$$
B(\omega)=\left\{f: T \rightarrow R^{\prime}, f(t)=\sum_{k=0}^{\infty} C_{k} b_{k}(t), \sum_{k=0}^{\infty}\left|C_{k}\right|<\infty\right\},
$$

each $b_{k}$ is a weighted special atom, that is, a real valued function $b$, defined on $T=[0,2 \pi]$, which is either $b(t)=1 /(2 \pi)$ or $b(t)=\omega(|Q|)^{-1 / q}$. $\left[\chi_{R}(t)-\chi_{L}(t)\right], 1 \leq q<\infty$, where $Q$ is an interval in $T, L$ is the left half of $Q$ and $R$ is the right half, $|Q|$ denotes the length of $Q, \chi_{Q}$ the characteristic function of $Q$ and $\omega$ is a non-negative real valued function which is increasing, and $\omega(0)=0 . \quad B(\omega)$ is endowed with the norm $\|f\|_{B(\omega)}=\inf \left\{\sum_{k=0}^{\infty}\left|C_{k}\right|\right\}$, where the infimum is taken over all possible representations of $f$. $B(\omega)$ is a Banach space.

A function $\omega:[0, \infty) \rightarrow[0, \infty)$ is said to be in the class $b_{\lambda}(0<\lambda<\infty)$, if it satisfies

(1) $\omega(0)=0$,

(2) $\omega$ is non-decreasing, $\omega(t) / t$ is decreasing,

(4) $\int_{0}^{h} \omega(t) / t d t \leq C \omega(h), C$ an absolute constant,

(5) $\int_{h}^{2 \pi} \omega(t) / t^{\lambda+1} d t \leq C \omega(h) / h^{\lambda}$ with $C$ independent of $h$ and $\omega$.

Example of functions in the class $b_{\lambda}$ are $\omega(t)=t^{\alpha} \quad(0<\alpha<1)$ and $\omega(t)=t^{\alpha}(\log (e / t))^{\beta}$, $(0<\alpha<\lambda, \beta \geq 0)$.

We also define the space $L(\phi)$ be $L(\phi)=\left\{f: T \rightarrow R^{\prime},\|f\|_{\phi}<\infty\right\}$, where $\|f\|_{\phi}=\left(\int_{T}\left(f^{*}(t)\right)^{q} \phi(t) d t\right)^{1 / q}, 1 \leq q<\infty$ and $f^{*}$ is the decreasing rearrangement of $f$, defined by $f^{*}(t)=\inf \{y:|\{x:|f(x)|>y\}| \leq t\}$, the outside bars means the Lebesgue measure of the set $\{x:|f(x)|>y\}, \phi$ is a non-negative decreasing function. $\|\cdot\|_{\phi}$ is a norm if and only if $\phi$ is a non-negative decreasing function. $L(\phi)$ is a Banach space. If $\omega(t)=(q / p) t^{q / p}, 1 \leq q \leq p<\infty$, $\phi(t)=\omega(t) / t$, then the space $L(\phi)$ is the Lorentz space $L(p, q)$ in $[6,7]$.

The main result of this paper is stated as follows:

THEOREM 1. Suppose $\omega \in b_{\lambda}, 1 \leq \lambda<\infty, \phi(t)=\omega(t) / t$, then $\sigma^{s}(f)$ is of type $(B(\omega), L(\phi))$ for all $s>0$, that is,

$$
\left\|\sigma^{s}(f)\right\|_{\phi} \leq C\|f\|_{B(\omega)}, \quad f \in B(\omega) .
$$

COROLLARY 1. Suppose $\omega \in b_{\lambda}, 1 \leq \lambda<\infty$, and $f \in B(\omega)$, then $\sigma_{r}^{s}(f, x)$ converges to $f(x)$ almost everywhere for all $s>0$.

THEOREM 2. Suppose $\omega \in b_{\lambda}, 1 \leq \lambda<\infty, \phi(t)=\omega(t) / t$, then $\tilde{\sigma}^{s}(f)$ is of type $(B(\omega), L(\phi))$ for all $s>0$, that is,

$$
\left\|\tilde{\sigma}^{s}(f)\right\|_{\phi} \leq C\|\tilde{f}\|_{B(\omega)} \leq C\|f\|_{B(\omega)}, \quad f \in B(\omega) .
$$

COROLLARY 2. Suppose $\omega \in b_{\lambda}, 1 \leq \lambda<\infty$, and $f \in B(\omega)$, then $\tilde{\sigma}_{r}^{s}(f, x)$ converges to $\tilde{f}(x)$ almost everywhere for all $s>0$.

REMARK 1. When $n=1, A(\xi)=|\xi|^{2}, \sigma_{r}^{s}(f, x)$ become

$$
\sigma_{r}^{s}(f, x)=\sum_{|k|<r}\left(1-(|k| / r)^{2}\right)^{s} \widehat{f}(k) e^{i k x} .
$$

As $s \rightarrow 0$, (1.3) become the partial sums of Fourier series of $f$, when $s=1 / 2,(1.3)$ are essentially equivalent to the classical Cesàro means. Consequently, the main result in $[5,6]$ become a special case of our results.

REMARK 2. For the maximal $(C, \alpha)$ operators $T$ are defined by

$$
T(f, x)=\sup _{n}\left|\sigma_{n}^{\alpha}(f, x)\right|
$$

where 


$$
\sigma_{n}^{\alpha}(f, x)=\frac{1}{\pi} \int_{-\pi}^{\pi} f(t) K_{n}^{\alpha}(x-t) d t
$$

since $(C, \alpha)$ kernels

$$
K_{n}^{\alpha}(t)=\sum_{k=0}^{n} A_{n-k}^{\alpha-1} D_{k}(t) / A_{n}^{\alpha}
$$

satisfies

$$
\left|K_{n}^{\alpha}(t)\right| \leq \begin{cases}\frac{A n}{(1+n t)\left(1+(n t)^{\alpha}\right)} \leq \frac{A n}{1+n t}, & 0<\alpha<1,0 \leq t \leq \pi \\ C /|t|, & \alpha=1,0<|t| \leq \pi\end{cases}
$$

thus using the same methods for $\phi(t)=\omega(t) / t$ we can prove

$$
\|T f\|_{\phi} \leq C\|f\|_{B(\omega)}, \quad f \in B(\omega), \quad 0<\alpha \leq 1 .
$$

\section{PROOFS OF THEOREMS}

PROOF OF THEOREM 1. Let $f^{\alpha}(x)=f(x-a)$, then the operator $T_{a} f=f^{a}$ is of type $(B(\omega), B(\omega))$. Consequently, we just need to prove the result for $f_{h}(t)=$ $[\omega(2 h)]^{-1 / q}\left[\chi_{[-h, 0)}(t)-\chi_{[0, h]}(t)\right], h>0$ which will follow from the estimate for $g(t)=\chi_{[0, h]}(t)$. Let $H(x)=(2 \pi)^{-1} \int_{R^{1}}(1-A(y))_{+}^{s} e^{2 x y} d y, s>0, \quad H_{1 / r}(x)=r H(r x)$, then $\sigma_{r}^{s}(f, x)=\left(f * K_{1 / r}\right)(x)$, where

$$
K_{1 / r}(x)=\sum_{k=-\infty}^{\infty} H_{1 / r}(x+2 k \pi)
$$

We may assume $r>1$. By the inequality (see [2]):

$$
|H(x)| \leq C(1+|x|)^{-s-1},
$$

we get

$$
\left|K_{1 / r}(x)\right| \leq C r \sum_{k=-\infty}^{\infty}(1+r|2 k \pi+x|)^{-(s+1)} \leq C r(1+r|x|)^{-(s+1)} .
$$

Thus

$$
\begin{aligned}
\left|\sigma_{r}^{s}(g, x)\right| & =\left|\int_{-\pi}^{\pi} g(y) K_{1 / r}(x-y) d y\right|=\left|\int_{0}^{h} K_{1 / r}(x-y) d y\right| \leq \int_{x-h}^{x}\left|K_{1 / r}(t)\right| d t \\
& \leq C \int_{x-h}^{x} r(1+r t)^{-1} d t \leq C h(x-h)^{-1}<2 C h / x
\end{aligned}
$$

for $x>2 h$, and $\left|\sigma_{r}^{s}(g, x)\right| \leq-2 C h / x$ for $x<-2 h$. On the other hand, we have

$$
\begin{aligned}
\left|\sigma_{r}^{s}(g, x)\right| & \leq \int_{0}^{h}\left|K_{1 / r}(x-y)\right| d y \leq \int_{-\pi}^{\pi}\left(\sum_{k=-\infty}^{\infty}\left|H_{1 / r}(x+2 k \pi-y)\right|\right) d y \\
& =\int_{-\infty}^{\infty}\left|H_{1 / r}(y)\right| d y \leq C \int_{-\infty}^{\infty}(1+|t|)^{-(s+1)} d t<\infty .
\end{aligned}
$$

Consequently, we have

$$
\left|\sigma^{s}(g, x)\right| \leq \begin{cases}A, & \text { for all } x, \\ 2 C h /|x|, & \text { for }|x|>2 h\end{cases}
$$

Let $\phi(t)=\omega(t) / t$. By (2.1) and the conditions on $\omega$, we get 


$$
\begin{aligned}
\left\|\sigma^{s}(g)\right\|_{\phi}^{q} & =\int_{0}^{2 \pi}\left(\left(\sigma^{s}(g)\right)^{*}(x)\right)^{q} \omega(x) / x d x \leq A^{q} \int_{0}^{2 h} \omega(x) / x d x \\
& +(2 C h)^{q} \int_{2 h}^{2 \pi} \omega(x) / x^{(q+1)} d x \leq C A^{q} \omega(2 h)+(2 C h)^{q}\left(\omega(2 h) /(2 h)^{q}\right)=C \omega(2 h) .
\end{aligned}
$$

The constant $C$ may not be the same at every occurrence in this paper. Thus $\left\|\sigma^{s}\left(f_{h}\right)\right\|_{\phi} \leq 2 \omega(2 h)^{-(1 / q)} \cdot\left\|\sigma^{s}(g)\right\|_{\phi} \leq C$ and so if $f \in B(\omega)$, then $f(t)=\sum_{k=0}^{\infty} C_{k} b_{k}(t)$, where

$$
b_{k}(t)=\omega\left(\left|Q_{k}\right|\right)^{-1 / q}\left[\chi_{R_{k}}(t)-\chi_{L_{k}}(t)\right]
$$

and $\sum_{k=0}^{\infty}\left|C_{k}\right|<\infty$, we have $\left\|\sigma^{s}(f)\right\|_{\phi} \leq C \sum_{k=0}^{\infty}\left|C_{k}\right|$, which implies $\left\|\sigma^{s}(f)\right\|_{\phi} \leq C\|f\|_{B(\omega)}$. The proof is complete.

\section{PROOF OF COROLLARY 1. Let}

then $\omega(f, x) \leq 2 \sigma^{s}(f, x)$ and so

$$
\omega(f, x)=\limsup _{r \rightarrow \infty}\left|\sigma_{r_{1}}^{s}(f, x)-\sigma_{r_{2}}^{s}(f, x)\right|, r_{1}, r_{2}>r,
$$

$$
\begin{aligned}
\|\omega(f)\|_{\phi}^{q} & =\int_{0}^{2 \pi}\left((\omega(f))^{*}(x)\right)^{q} \omega(x) / x d x \leq 2 \int_{0}^{2 \pi}\left(\left(\sigma^{s}(f)\right)^{*}(x)\right)^{q} \omega(x) / x d x \\
& =2\left\|\sigma^{s}(f)\right\|_{\phi}^{q} .
\end{aligned}
$$

Since $f \in B(\omega)$, then $f(x)=\sum_{k=0}^{\infty} C_{k} b_{k}(x)$, where $\sum_{k=0}^{\infty}\left|C_{k}\right|<\infty$ and the $b_{k}$ are weighted special atoms. By Theorem 1 and (2.3), $\sigma^{s}(f) \in L(\phi)$ which implies $\omega(f) \in L(\phi)$ for $\phi(t)=\omega(t) / t$. On the other hand, we see that $\omega(f)=\omega\left(f-f_{m}\right)$ where $f_{m}(x)=\sum_{k=0}^{m} C_{k} b_{k}(x)$ and $\left\|f_{m}-f\right\|_{B(\omega)} \rightarrow 0$ as $m \rightarrow \infty$. Then

$$
\omega(f, x)=\omega\left(f-f_{m}, x\right) \leq 2 \sigma^{s}\left(f-f_{m}, x\right) .
$$

By Theorem 1,

$$
\|\omega(f)\|_{\phi} \leq 2\left\|\sigma^{s}\left(f-f_{m}\right)\right\|_{\phi} \leq 2 C\left\|f-f_{m}\right\|_{B(\omega)} .
$$

So letting $m \rightarrow \infty$, we get $\|\omega(f)\|_{\phi}=0$. Thus $\omega(f, x)=0$ almost everywhere, which implies $\sigma_{r}^{s}(f, x)$ converges to $f(x)$ almost everywhere. The proof is complete.

Let $f \in B(\omega)$, then $f(x)=\sum_{k=0}^{\infty} C_{k} b_{k}(x)$, where $\sum_{k=0}^{\infty}\left|C_{k}\right|<\infty$ and the $b_{k}$ are weighted special atoms. Thus $\tilde{f}(x)=\sum_{k=0}^{\infty} C_{k} \tilde{b}_{k}(x)$ and so $\|\tilde{f}\|_{B(\omega)} \leq\|f\|_{B(\omega)}$. Now using $\tilde{\sigma}_{r}^{s}(f, x)=\sigma_{r}^{s}(\tilde{f}, x)$ and Theorem 1, we can similarly show that Theorem 2 and Corollary 2 . The details will be omitted.

REMARK 3. Theorem 1 and 2 are also true if we replace the above $\omega(|Q|)$ by a weight $\omega(Q)=\int_{Q} \omega(x) d x$, where $\omega$ in $A_{\infty}$, the proofs are the same.

\section{REFERENCES}

[1] LU, S.Z. and WANG, K.Y., Bochner-Reisz Means, Beijing, 1989.

[2] JANG, Y.S., LIU, H.P. and LU, S.Z., Some properties of elliptic Riesz means at critical index on $H^{p}\left(T^{n}\right)$, Approx. Theory \& Its Appl. 6 (1990), 2, 28-37.

[3] KUANG JCHANG, Approximation of elliptic Riesz operators on $H^{p}\left(T^{n}\right)$, Acta Sci. Nat. Univ. Norm. Hunan 12 (1989), 1, 1-6, MR90g:41052.

[4] BLOOM, S. and DeSOUZA, G.S., Atomic decomposition of the generalized Lipschitz spaces, Illinois J. of Math. 33 (1989), 2, 181-209.

[5] DeSOUZA, G.S., Fourier series and the maximal operator on the weighted special atom spaces, Internat. J. Math. and Math. Sci. 12 (1989), 3, 579-582.

[6] DeSOUZA, G.S., On the convergence of Fourier series, Internat. J. Math and Math. Sci. 7 (4) (1984), 817-820.

[7] LORENTZ, G.G., Some new functional spaces, Ann. of Math. 51 (1950), 37-55. 


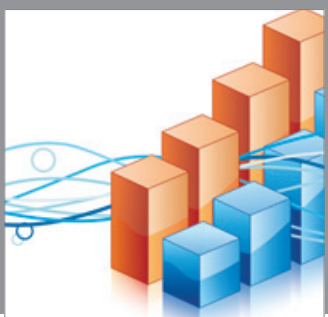

Advances in

Operations Research

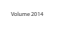

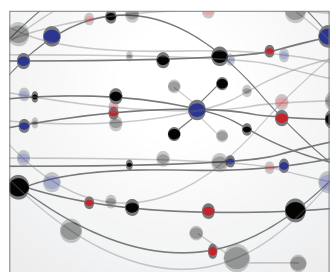

\section{The Scientific} World Journal
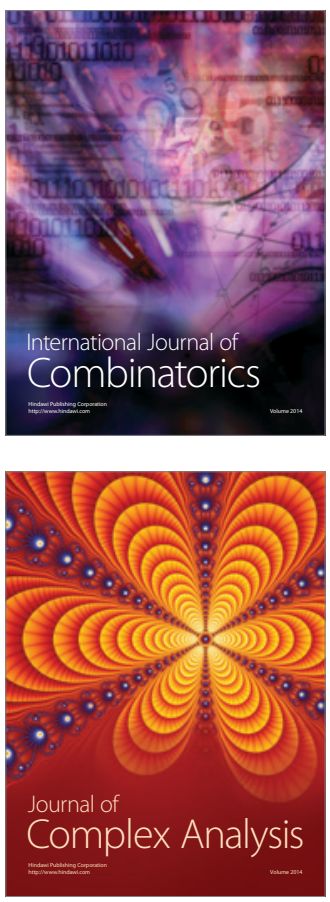

International Journal of

Mathematics and

Mathematical

Sciences
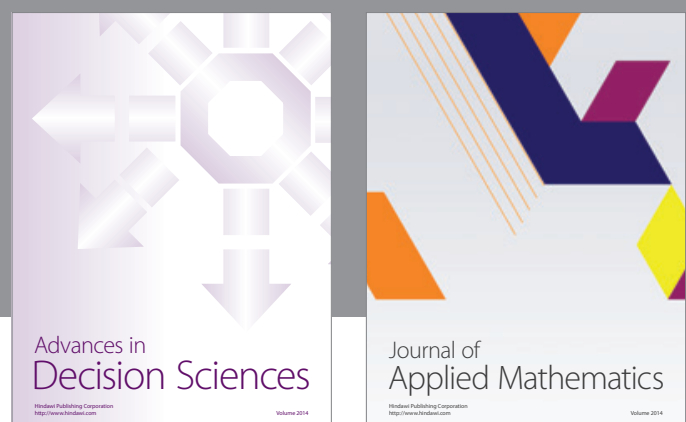

Journal of

Applied Mathematics
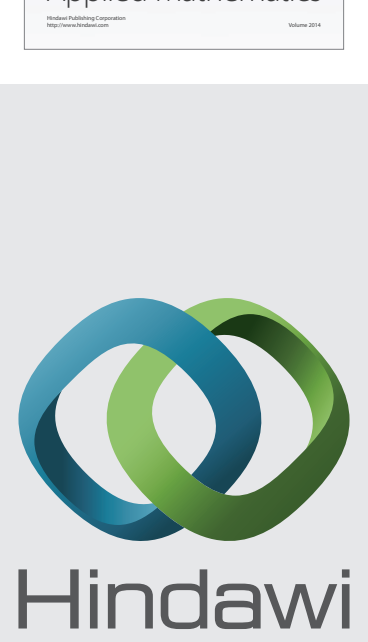

Submit your manuscripts at http://www.hindawi.com
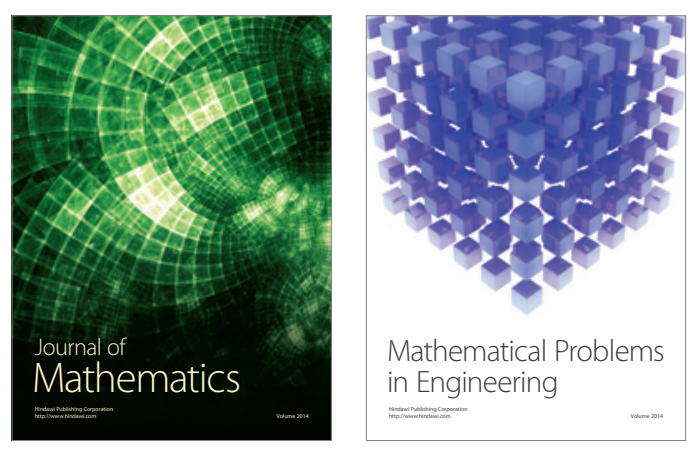

Mathematical Problems in Engineering
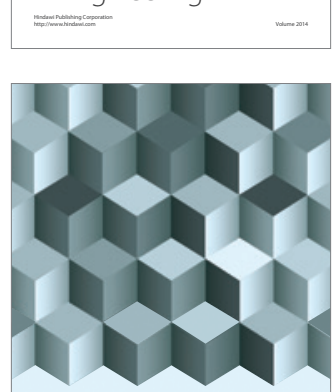

Journal of

Function Spaces
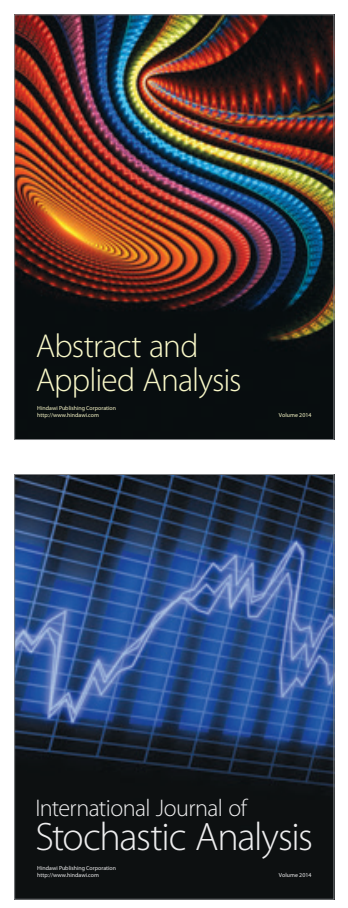

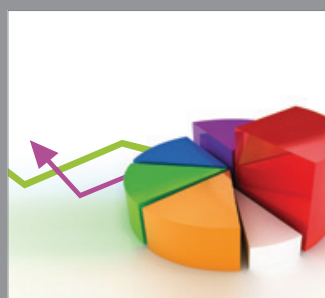

ournal of

Probability and Statistics

Promensencen
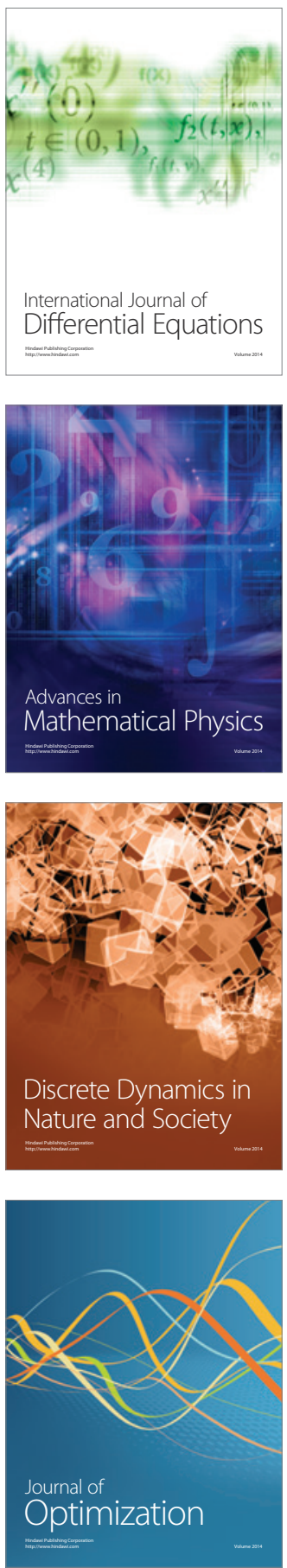